\title{
LncRNA AWPPH participates in the metastasis of non-small cell lung cancer by upregulating TGF- $\beta 1$ expression
}

\author{
YANXIA HUO, AIMIN LI and ZHIHUA WANG \\ Department of Respiratory Medicine, Affiliated Xing Tai People Hospital of Hebei Medical University, \\ Xingtai, Hebei 054031, P.R. China
}

Received October 30, 2018; Accepted July 26, 2019

DOI: 10.3892/ol.2019.10754

\begin{abstract}
Long non-coding RNA (lncRNA) AWPPH is a characterized oncogenic lncRNA in hepatocellular carcinoma and bladder cancer. The aim of the present study was to investigate the potential involvement of AWPPH in non-small cell lung cancer (NSCLC). Expression of AWPPH in lung biopsy tissues and serum of both healthy controls and patients with NSCLC with different tumor sizes, and with and without distant tumor metastasis was detected by reverse transcription-quantitative PCR. AWPPH expression vector was constructed and transfected into cells of human NSCLC cell lines. Expression of TGF- $\beta 1$ was detected by western blot analysis. Cell migration and invasion were detected by Transwell migration and invasion assay. It was observed that AWPPH was significantly upregulated in patients with NSCLC, while AWPPH expression level did not increase with the increase of tumor size. In contrast, AWPPH expression levels were significantly higher in patients with distant metastasis than in patients without tumor distant metastasis. AWPPH overexpression promoted TGF- $\beta 1$ expression in NSCLC cells, while TGF- $\beta 1$ treatment showed no significant effects on AWPPH expression. AWPPH overexpression promoted NSCLC cell migration and invasion, while TGF- $\beta$ signaling inhibition reduced this enhancing effect. Therefore, AWPPH may promote the metastasis, but not the growth of NSCLC by upregulating TGF- $\beta 1$ expression.
\end{abstract}

\section{Introduction}

Lung cancer is the most common and deadly malignancies worldwide (1). As a heterogeneous class of malignant tumors, non-small-cell lung cancer (NSCLC) accounts for $85 \%$ of lung cancer cases (2). Tobacco consumption remains the main risk

Correspondence to: Dr Aimin Li, Department of Respiratory Medicine, Affiliated Xing Tai People Hospital of Hebei Medical University, 16 Red Star Street, Xingtai, Hebei 054031, P.R. China E-mail: ukqar02@163.com

Key words: long non-coding RNA AWPPH, non-small cell lung cancer, growth, metastasis factor of NSCLC, however studies in recent years have shown that air pollution and radon exposure also contribute to the development of this disease (3). Incidence of NSCLC in recent years has shown an increased trend in developing countries, such as China (4). The majority of patients with NSCLC were diagnosed at advanced stages with distant tumor metastasis, which has been reported as the main cause of death (5). Therefore, early detection of tumor distant metastasis remains crucial for the treatment of NSCLC.

Metastasis of NSCLC requires the involvement of multiple signaling pathways $(6,7)$, in which TGF- $\beta$ signaling transduction plays a central role (6). Activation of TGF- $\beta$ signal in NSCLC tissues promotes epithelial mesenchymal transition, thereby inducing the invasion and migration of cancer cells (8). Long (>200 nt) non-coding RNAs, or lncRNAs, are a group of non-protein coding RNA transcripts with oncogenic or tumor suppression functions in cancer development (9). The cross-talks between IncRNAs and TGF- $\beta$ pathway have been reported (10). LncRNA AWPPH is a novel lncRNA with the oncogenic role in hepatocellular carcinoma (11), bladder cancer (12) and triple-negative breast cancer (13). AWPPH is located on chromosome 2 and is the host of MIR4435-2 (12). In hepatocellular carcinoma, lncRNA AWPPH interacts with Y-box binding protein 1 to promote disease progression (11). In bladder cancer, lncRNA AWPPH regulates proliferation, autophagy, and migration, but inhibits apoptosis of bladder cancer cells by inhibiting SMAD4 via enhancer of zeste 2 polycomb repressive complex 2 subunit (12). However, to the best of our knowledge, the involvement of AWPPH in NSCLC and its interactions with TGF- $\beta$ signaling remains unknown. In the present study, it was observed that lncRNA AWPPH may promote the metastasis, but not the growth of NSCLC by upregulating TGF- $\beta 1$ expression.

\section{Materials and methods}

Patients. This study included 138 patients with NSCLC, who were pathologically diagnosed at the Affiliated Xing Tai People Hospital of Hebei Medical University between January 2015 and January 2018. Inclusion criteria were as follows: Patients pathologically diagnosed with NSCLC; new NSCLC cases and patients with normal functions of other major organs. Exclusion criteria were as follows: Patients suffering from other malignancies; patients with other lung diseases; patients 
with mental disorders and patients with an education level below elementary high school that would inhibit cooperation with researchers. This study's patients included 88 males and 50 females, and the age ranged between 26 and 72 years, with a mean age of $50 \pm 5.6$ years. According to computed tomography scanning results, 28 patients presented with a primary tumor diameter between 0 and $1 \mathrm{~cm}, 21$ patients were between 1 and $2 \mathrm{~cm}, 25$ patients were between 2 and $3 \mathrm{~cm}, 20$ patients were between 3 and $4 \mathrm{~cm}, 23$ patients were between 4 and $5 \mathrm{~cm}$, and 21 patients were $>5 \mathrm{~cm}$. Tumor metastasis was observed in 72 patients, including 14 cases of brain metastasis, 13 cases of bone metastasis, 20 cases of liver metastasis and 25 cases of lymphatic metastasis. Tumor metastasis was not found in the remaining 66 patients. At the same time, 32 healthy volunteers, including 20 male and 12 female patients (range, 29-69 years, mean 48.3 \pm 5.1 years) served as the control group. Control group, different tumor size groups and different metastasis groups showed similar sex and age distributions. The Ethics Committee of Affiliated Xing Tai People Hospital of Hebei Medical University approved the present study and all participants signed an informed consent.

Specimen collection. Lung biopsies were obtained from patients with NSCLC and healthy controls. Healthy controls received biopsies to detect potential lung lesions, however any suspected lung diseases were excluded. Blood was also extracted from each participant on the day of admission. Blood samples were stored at room temperature for $20 \mathrm{~min}$ and were centrifuged at $1,200 \mathrm{x}$ g for $20 \mathrm{~min}$ at room temperature to collect serum. All specimens were stored in liquid nitrogen before use.

ELISA. TGF- $\beta 1$ in serum was detected using a human TGF- $\beta 1$ ELISA kit (cat. no., ab100647, Abcam), according to the manufacturer's protocols. Plasma levels of TGF- $\beta 1$ were normalized to $\mathrm{ng} / \mathrm{ml}$.

Reverse transcription-quantitative PCR (RT-qPCR). Total RNA was extracted from in vitro cultvated cells, biopsies and plasma using TRIzol reagent (Sigma-Aldrich; Merck KGaA) and subjected to reverse transcription to synthesize cDNA. In cases of TGF- $\beta 1$ treatment, cells were cultviated in medium containing 5, 10 and $20 \mathrm{ng} / \mathrm{ml} \mathrm{TGF- \beta 1} \mathrm{(Abcam)} \mathrm{under} \mathrm{afore-}$ mentioned conditions for $24 \mathrm{~h}$ prior to use. PCR reaction was performed using SYBR-Green PCR Master Mix (Thermo Fisher Scientific, Inc.), using the primers: 5'-CTGGATGGT CGCTGCTTTTTA-3' (forward) and 5'-AGGGGGATGAGT CGTGATTT-3' (reverse) for human lncRNA AWPPH (11); 5'-GACCTCTATGCCAACACAGT3' (forward) and 5'-AGT ACTTGCGCTCAGGAGGA3' (reverse) for $\beta$-actin. The thermocycling PCR reaction conditions were as follows: $95^{\circ} \mathrm{C}$ for $1 \mathrm{~min}, 40$ cycles of $95^{\circ} \mathrm{C}$ for $10 \mathrm{sec}$ and $56^{\circ} \mathrm{C}$ for $20 \mathrm{sec} . \mathrm{Cq}$ values were processed using $2^{-\Delta \Delta \mathrm{Cq}}$ method (14) and AWPPH expression was normalized to endogenous control $\beta$-actin.

Cell line, cell culture and transfection. Normal lung epithelial cell line, NuLi-1, and human NSCLC cell lines NCI-H1581 (H1581), as well as NCI-H1993 (H1993), were purchased from the American Type Culture Collection (ATCC). RPMI-1640 medium (ATCC) containing 10\% fetal bovine serum (FBS; cat. no., ATCC 30-2020; ATCC) was used as cell culture medium and cell culture conditions were at $37^{\circ} \mathrm{C}$ with $5 \% \mathrm{CO}_{2}$. Full-length AWPPH cDNA (accession no.: NR_015395.2) was inserted into pIRSE2-EGFP vector (Clontech Laboratories, Inc.) to make AWPPH expression vector. AWPPH short hairpin (sh)RNA (GGTCTGGTCGGTTTCCCATTT) and scrambled shControl (TCCTAAGGTTAAGTCGCCCTC) were synthesized by GenePharma, Inc. AWPPH expression vectors (10 nM) and shRNA (20 nM) were transfected into 6x10 $0^{5}$ cells using Lipofectamine 2000 reagent (cat. no., 11668-019; Invitrogen; Thermo Fisher Scientific, Inc.). Empty vector transfection and transfection with scrambled shControl were also performed to serve as negative controls. Cells without transfection were used as controls. Overexpression and knockdown were confirmed by RT-qPCR before subsequent experiments. Subsequent experiments were performed when the overexpression and knockdown rates were $>200$ and $<50 \%$, respectively, compared with control cells. The interval between transfection and following experiments was $24 \mathrm{~h}$.

Transwell migration and invasion assay. Cells were collected by centrifugation at $1,200 \mathrm{x}$ for $10 \mathrm{~min}$ at room temperature and mixed with RPMI-1640 medium (1\% FBS) to prepare single cell suspesions $\left(4 \times 10^{4}\right.$ cells $\left./ \mathrm{ml}\right)$. In cases of TGF- $\beta$ signaling inhibition, cells were treated with TGF- $\beta$ receptor inhibitor SB431542 (SB; 10 nM, Sigma-Aldrich; Merck $\mathrm{KGaA}$ ) at $37^{\circ} \mathrm{C}$ for $24 \mathrm{~h}$ before use. Regarding migration assay, $0.1 \mathrm{ml}$ cell suspension (non-serum RPMI-1640 medium) were added to the upper Transwell Inserts (Corning), while the low Transwell chamber was filled with RPMI-1640 medium containing 20\% FBS. After cell culture for $24 \mathrm{~h}$, Transwell chamber membranes were cleaned and stained with $0.5 \%$ crystal violet (Sigma-Aldrich; Merck $\mathrm{KGaA}$ ) for $15 \mathrm{~min}$ at $22^{\circ} \mathrm{C}$. For the invasion assay the upper transwell chamber was precoated with Matrigel (cat. no., 356234; EMD Millipore) before experiments. Cell migration and invasion were observed under an optical microscope (magnification, $\mathrm{x} 40$ ). Cell migration and invasion rates were normalized to the cell proliferation rate at the same time point.

Westernblotanalysis.RIPA solution(Thermo Fisher Scientific, Inc.) was used to extract protein and protein concentration was determined through BCA assay (Sigma-Aldrich; Merck $\mathrm{KGaA})$. Protein $(20 \mu \mathrm{g})$ was mixed with loading buffer, denatured and added into each lane of $10 \%$ SDS-PAGE gel. After electrophoresis, proteins transferred to PVDF membranes were blocked with $5 \%$ skimmed milk at $22^{\circ} \mathrm{C}$ for $1 \mathrm{~h}$, followed by incubation with TGF- $\beta 1$ (cat. no., ab9758; dilution 1:1,200; Abcam) GAPDH (rabbit anti human; cat. no., ab9485; dilution, 1:1,400; Abcam) rabbit anti-human primary antibodies at $4^{\circ} \mathrm{C}$ overnight. The next day, the membranes were washed with TBST (0.3\% Tween-20), followed by incubation with IgG-HRP (dilution, 1:1,000; cat. no., MBS435036; MyBioSource, Inc.) secondary antibody at room temperature for $3 \mathrm{~h}$. Immobilon ${ }^{\circledR}$ ECL Ultra Western HRP Substrate (cat. no., WBULS0100; Sigma-Aldrich; Merck KGaA) was subsequently spread onto the membranes to develop signals, and the grey band of TGF- $\beta 1$ was normalized to that of GAPDH using Image J v.148 software (National Institutes of Health, Bethesda, MD, USA). 
A

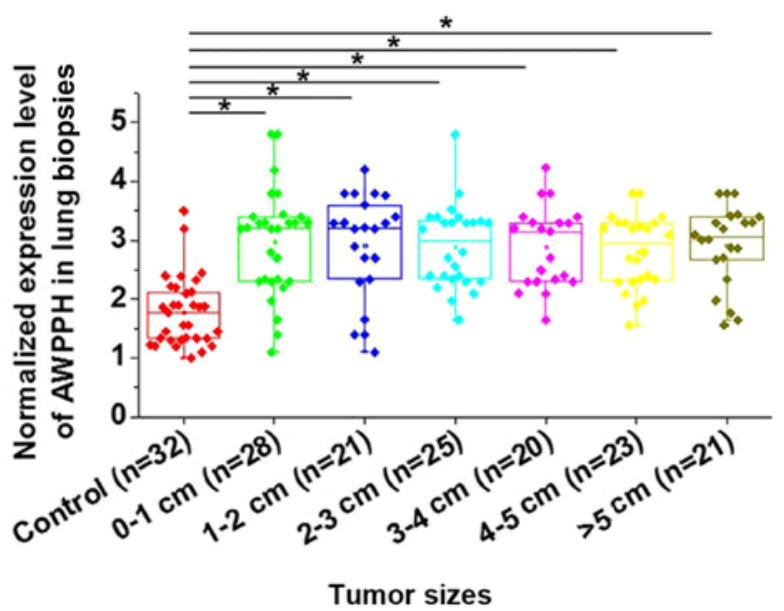

B

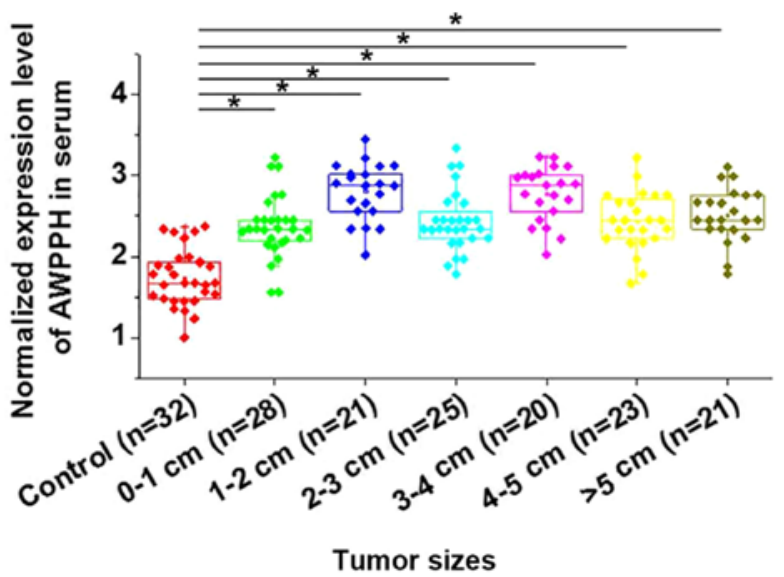

Figure 1. Expression of AWPPH in lung biopsies and serum of healthy controls and patients with different tumor sizes. Patients were divided into different tumor size groups, according to the largest tumor diameter. Normalized expression levels of AWPPH in (A) lung biopsies and (B) serum of healthy controls and patients with different tumor sizes. $\mathrm{P}<0.05$.

Statistical analysis. Graphpad Prism 6 software (GraphPad Software, Inc.) was used. Data were expressed as mean \pm standard deviation. Three biological replicates were included in each experiment. Mean values were compared by one-way ANOVA and Tukey's post hoc test. Correlations were analyzed by linear regression. $\mathrm{P}<0.05$ was considered to indicate a statistically significant difference.

\section{Results}

Expression of AWPPH in lung biopsies and serum of healthy controls and patients with different tumor sizes. As shown in Fig. 1, expression of AWPPH was found to be significantly upregulated in patients with NSCLC with different primary tumor diameters compared with healthy controls in lung biopsies (Fig. 1A) and serum (Fig. 1B) $(\mathrm{P}<0.05)$. However, no significant differences in AWPPH expression were found among patients with different tumor diameters. Correlations were analyzed by linear regression. It was observed that expression levels of AWPPH were significantly correlated with expression levels in serum $\left(\mathrm{R}^{2}=0.67 ; \mathrm{P}<0.0001\right)$. In addition, no significant differences in AWPPH expression were found
A

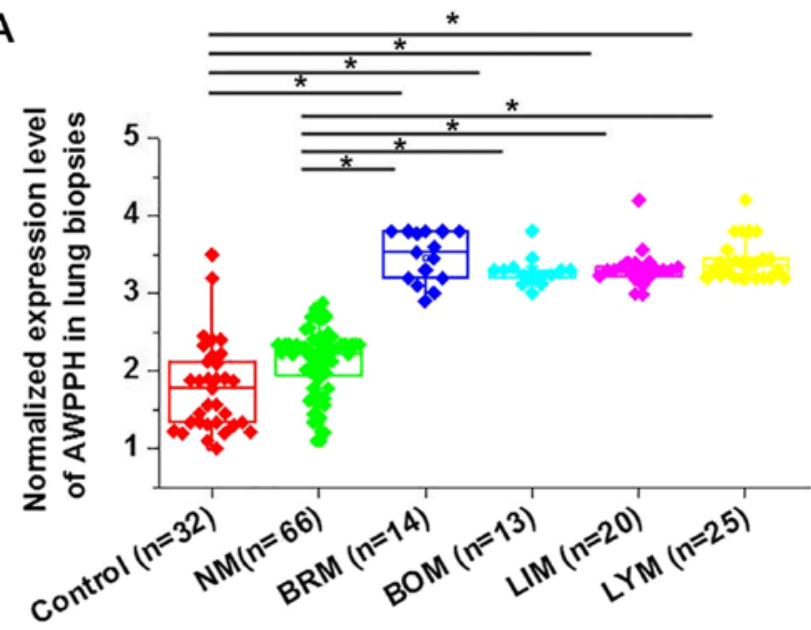

Tumor metastasis

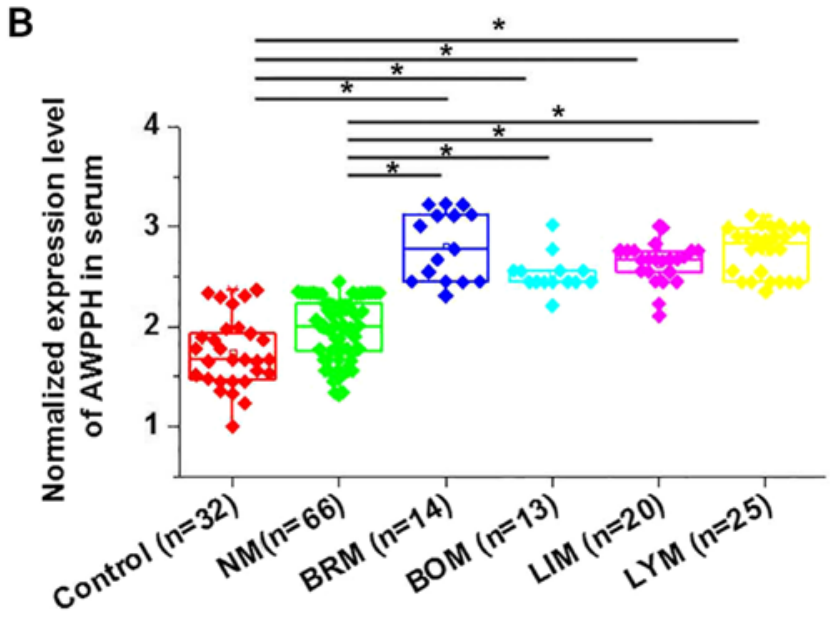

Tumor metastasis

Figure 2. Expression of AWPPH in lung biopsies and serum of healthy controls and patients with different metastasis status. Patients were divided into different tumor metastasis groups. Normalized expression levels of AWPPH in (A) lung biopsies and (B) serum of healthy controls and patients with different metastasis status. ${ }^{*} \mathrm{P}<0.05$. NM, non-metastasis; BRM, brain metastasis; BOM, bone metastasis; LIM, liver metastasis; LYM, lymphatic metastasis.

among patients with different $\mathrm{T}$ stages $(3 ; 3-5 ;>5 \mathrm{~cm}$; data not shown).

Expression of AWPPH in lung biopsies and serum of healthy controls and patients with different metastasis status. This study further compared the expression of AWPPH in lung biopsies and serum of healthy controls and patients with different metastasis status to investigate its potential involvement in cancer metastasis. As shown in Fig. 2, no significant differences in AWPPH expression in lung biopsies (Fig. 2A) and serum (Fig. 2B) were found between heathy controls and patients with NSCLC without metastasis. Significant upregulated expression of AWPPH in lung biopsies (Fig. 2A) and serum (Fig. 2B) were found in patients with 4 different types of cancer metastasis $(\mathrm{P}<0.05)$ compared to heathy controls and non-metastatic patients with NSCLC. Therefore, AWPPH is likely to be involved in the metastasis of NSCLC. 
A
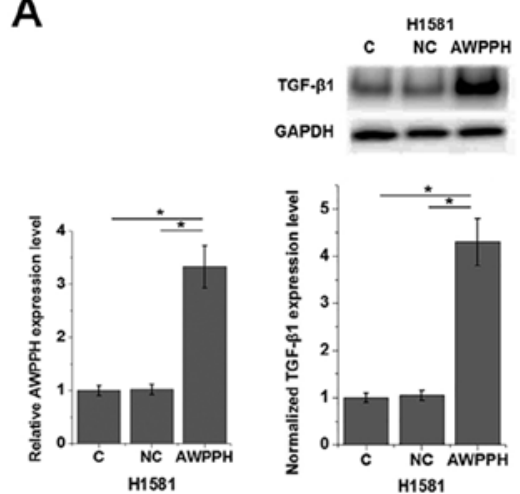

B
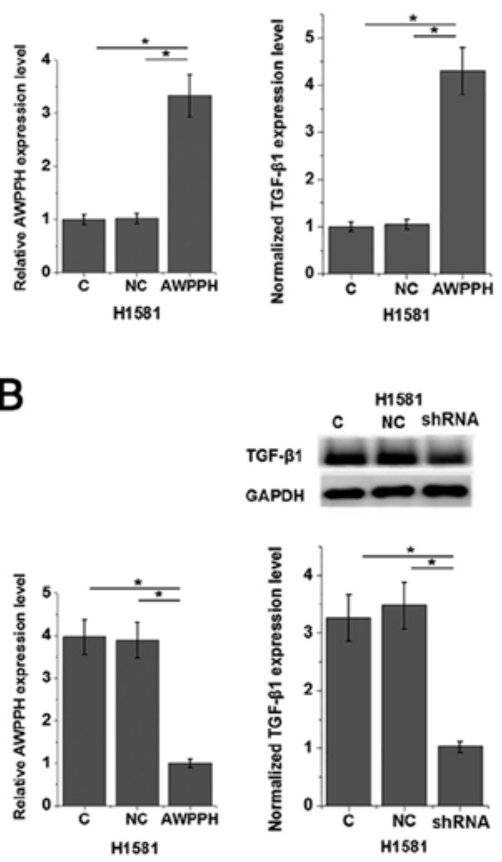

C

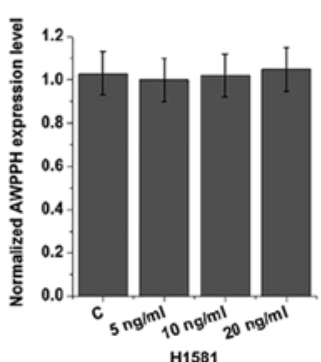

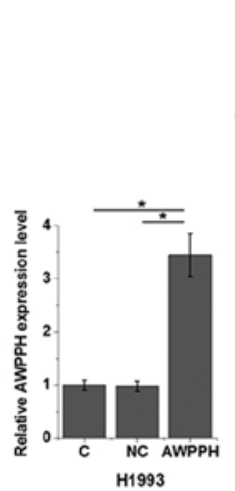
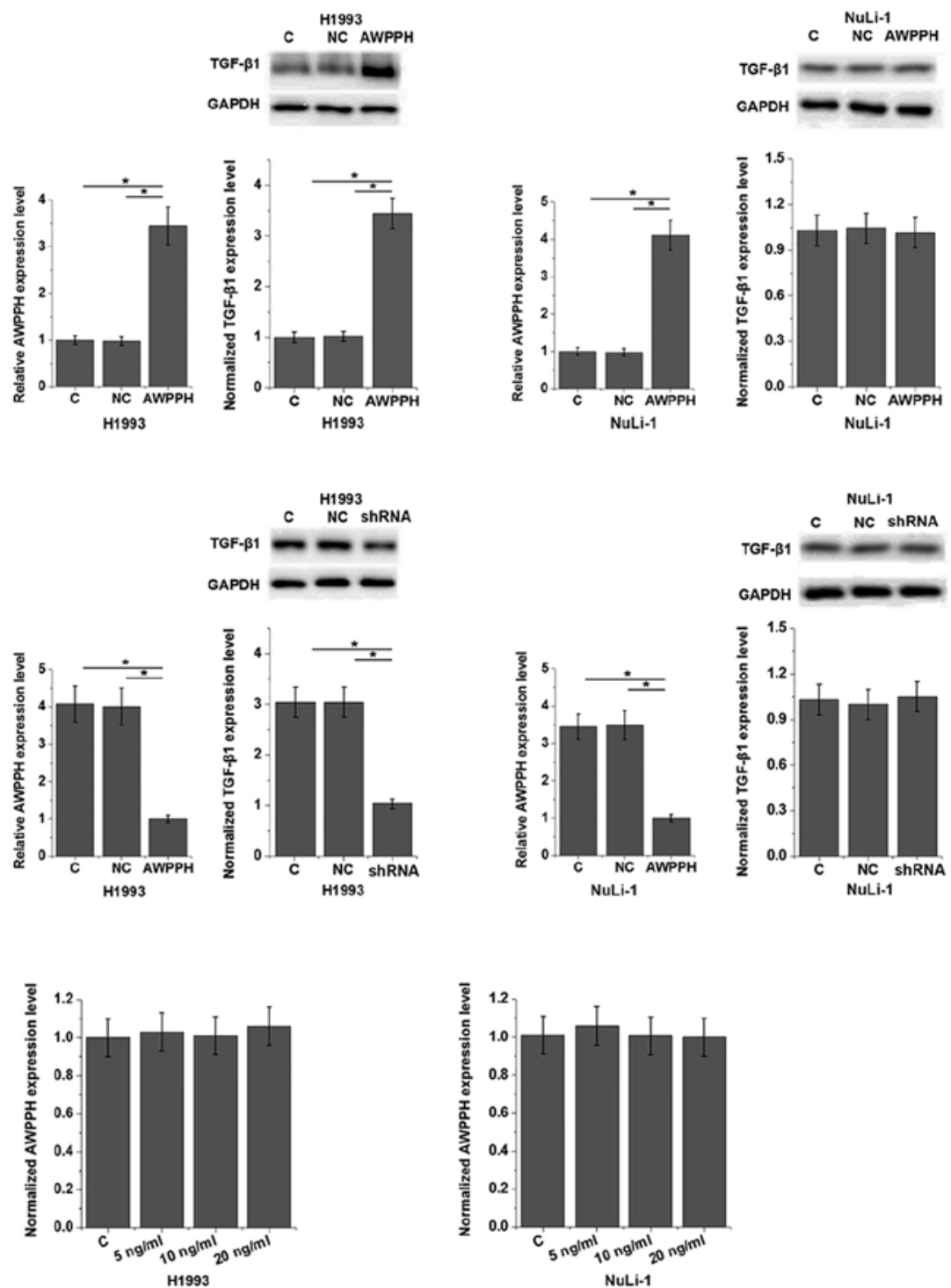

Figure 3. Potential associations between AWPPH and TGF- $\beta 1$ in NSCLC cells and normal lung cancer cells. Effects of AWPPH (A) overexpression and (B) knockdown on TGF- $\beta 1$ expression. NC cells were transfected with empty vector or scrambled shControl, respectively. (C) Effects of TGF- $\beta 1$ treatment on AWPPH expression in cells of normal human lung cell line NuLi-1 and human NSCLC cell lines H1581 and H1993. * $<0.05$. C, control cells without any treatment; NC, cells transfected with empty vector/scrambled shControl; NSCLC, non-small cell lung cancer; sh, short hairpin.

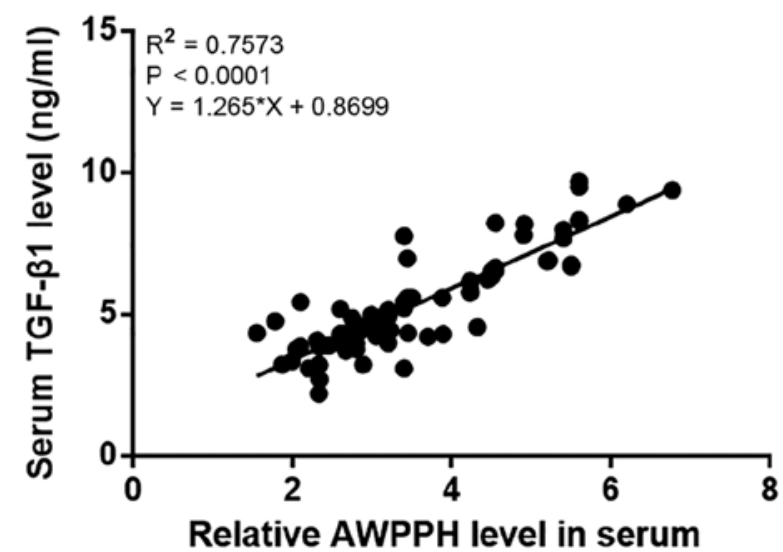

Figure 4. Serum TGF- $\beta 1$ and AWPPH indicated a positive correlation in NSCLC. Correlation between serum levels of TGF- $\beta 1$ and AWPPH in patients with NSCLC was analyzed by linear regression. It was observed that TGF- $\beta 1$ and AWPPH were significantly and positively correlated. NSCLC, non-small cell lung cancer.
Potential interactions between AWPPH and TGF- $\beta 1$ in NSCLC cells and normal lung cancer cells. TGF- $\beta$ signaling plays a pivotal role in the metastasis of different types of human malignancies, including lung cancer (6). Therefore, the potential interactions between AWPPH and TGF- $\beta 1$ were investigated. Analysis of AWPPH expression in cells of different cell lines showed that the expression level of AWPPH was significantly higher in cells of NSCLC cell lines H1581 (4.32-fold) and H1993 (3.37-fold) than in cells of the normal human lung cell line NuLi-1 (Fig. S1). As shown in Fig. 3A, AWPPH overexpression significantly promoted the expression of TGF- $\beta 1$ in the two human NSCLC cell lines, H1581 and H1993 $(\mathrm{P}<0.05)$, however not in NuLi-1 cells $(\mathrm{P}>0.05)$. By contrast, AWPPH-knockdown significantly inhibited the expression of TGF- $\beta 1$ in the two human NSCLC cell lines, H1581 and H1993 ( $<<0.05)$, but not in cells of the normal human lung cell line NuLi-1 (Fig. 3B; P>0.05). In addition, TGF- $\beta 1$ (Abcam) treatment at concentrations of 5, 10 and 
A
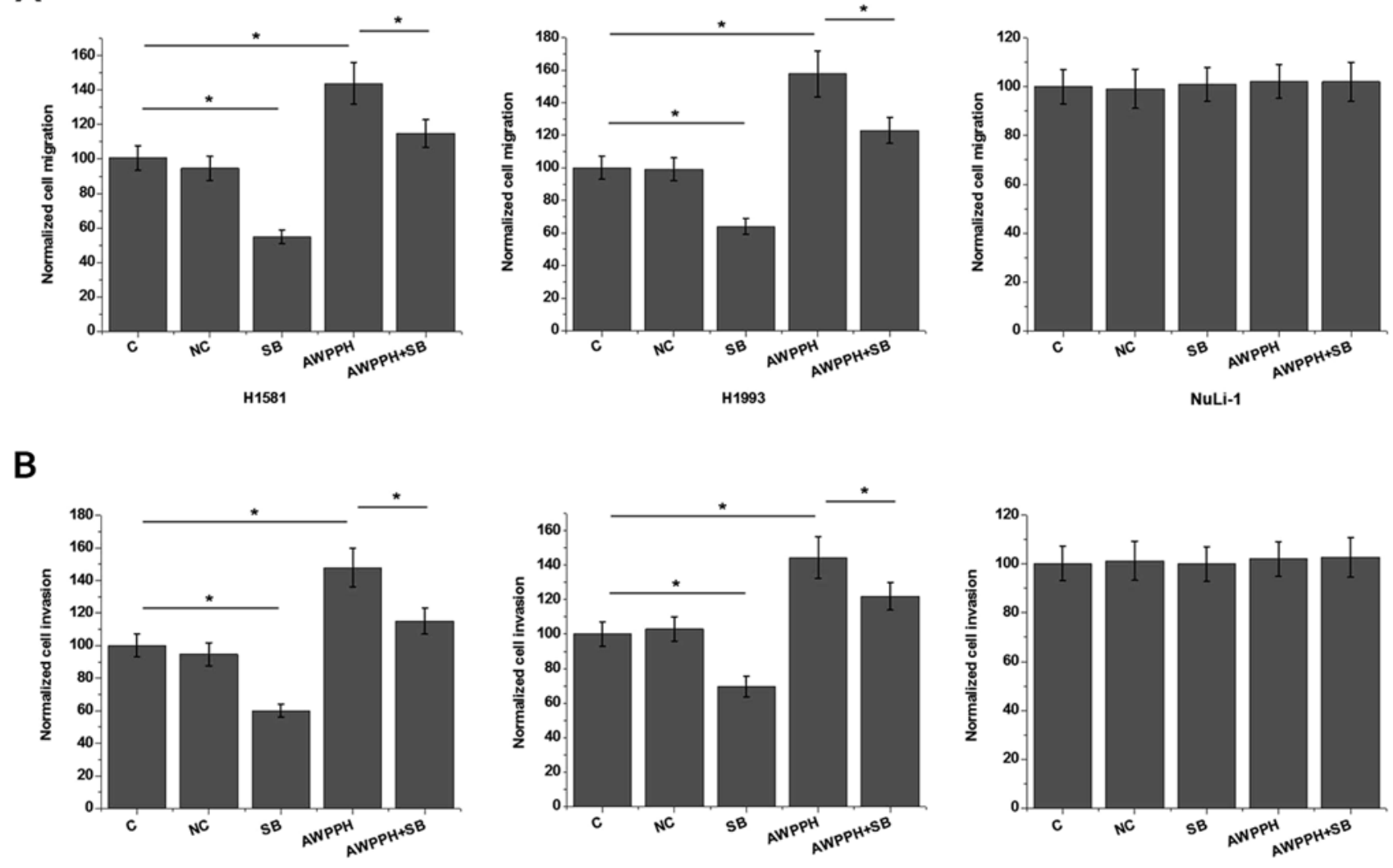

H1581

H1993

NuLi-1

Figure 5. Effects of AWPPH overexpression and TGF- $\beta$ inhibitor on cell migration and invasion. Effects of AWPPH overexpression and TGF- $\beta$ inhibitor treatment on (A) migration and (B) invasion of cells of normal human lung cell line NuLi-1 and human non-small cell lung cancer cell lines H1581 and H1993. ${ }^{*} \mathrm{P}<0.05$. C, control cells without any treatment; NC, cells transfected with empty vector; SB, SB431542.

$20 \mathrm{ng} / \mathrm{ml}$ showed no significant effects on AWPPH expression (Fig. 3C). Therefore, AWPPH is likely an upstream activator of TGF- $\beta$ signaling specifically in NSCLC cells, however not in normal lung cells. In addition, the correlation between serum levels of TGF- $\beta 1$ and AWPPH in patients with NSCLC was analyzed by linear regression. It was observed that TGF- $\beta 1$ and AWPPH were significantly and positively correlated $\left(\mathrm{R}^{2}=0.7573 ; \mathrm{P}<0.0001 ;\right.$ Fig. 4).

Effects of AWPPH overexpression and TGF- $\beta$ inhibition on cell migration and invasion. In vitro cell migration and invasion assay was performed to further investigate the involvement of AWPPH-TGF- $\beta 1$ signaling in the regulation of NSCLC metastasis. As showed in Fig. 4, AWPPH overexpression significantly promoted $(\mathrm{P}<0.05)$, while treatment with TGF- $\beta$ inhibitor SB431542 significantly inhibited the migration (Fig. 5A) and invasion (Fig. 5B) of the two human NSCLC cell lines $(\mathrm{P}<0.05)$. In addition, treatment with TGF- $\beta$ inhibitor SB431542 significantly reduced the enhancing effects of AWPPH overexpression on NSCLC migration (Fig. 4A) and invasion (Fig. 4B) $(\mathrm{P}<0.05)$. In addition, AWPPH overexpression and treatment with TGF- $\beta$ inhibitor SB431542 showed no significant effects on cells of the normal human lung cell line NuLi-1 ( $\mathrm{P}>0.05)$. By contrast, AWPPH shRNA-knockdown significantly inhibited the migration (Fig. 6A) and invasion (Fig. 6B) of the two human NSCLC cell lines $(\mathrm{P}<0.05)$.

\section{Discussion}

LncRNA AWPPH is a newly identified lncRNA with characterized oncogenic role in hepatocellular carcinoma (11), bladder cancer (12) and breast cancer (13). The main finding of this study indicated that AWPPH may participate in metastasis, but not growth of NSCLC. It was also found that the action of AWPPH in NSCLC is likely to be achieved through the upregulation of TGF- $\beta 1$.

Genetic alterations participate in NSCLC (15). Genetic alterations not only define the subtypes of NSCLC, but also provide guidance for therapeutic treatments (16). It has also been reported that the onset, development, progression and almost all pathological changes in NSCLC are accompanied with changes in the expression patterns of a large set of lncRNAs (17). The involvement and functionality of those lncRNAs in NSCLC have been gradually identified $(18,19)$. Upregulation of lncRNA PVT1 is frequently observed in patients with NSCLC, and overexpression of lncRNA plays a role as an oncogene to promote tumorigenesis in this disease (18). By contrast, lncRNA maternally expressed 3 (MEG3) was downregulated in NSCLC, and overexpression of this lncRNA inhibits cancer cell proliferation and induces cell apoptosis, indicating the potential application of MEG3 as a therapeutic target for NSCLC (19). Besides those two IncRNAs, functionality of a considerable number of IncRNAs has been characterized in NSCLC (16). However, the aforementioned 
A
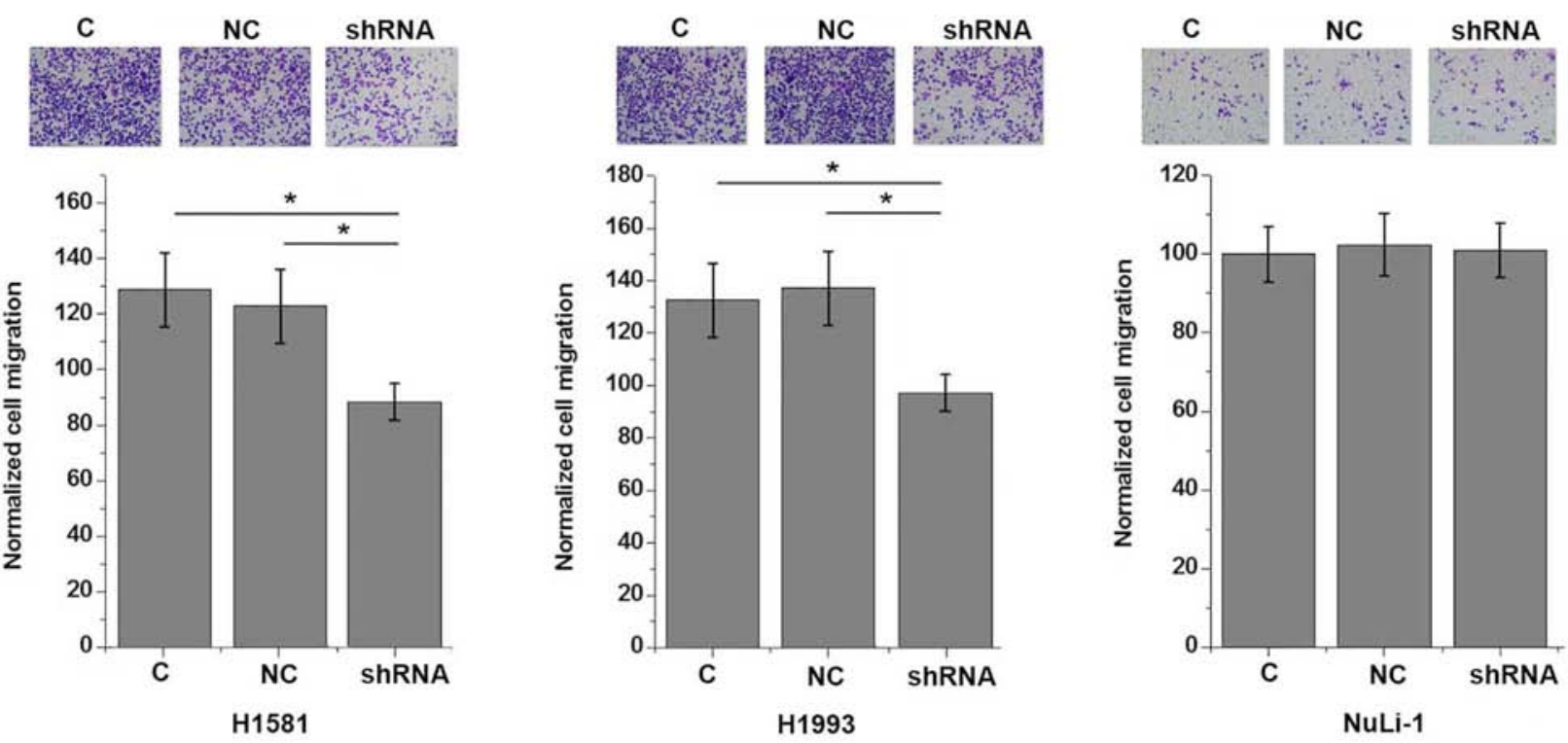

B
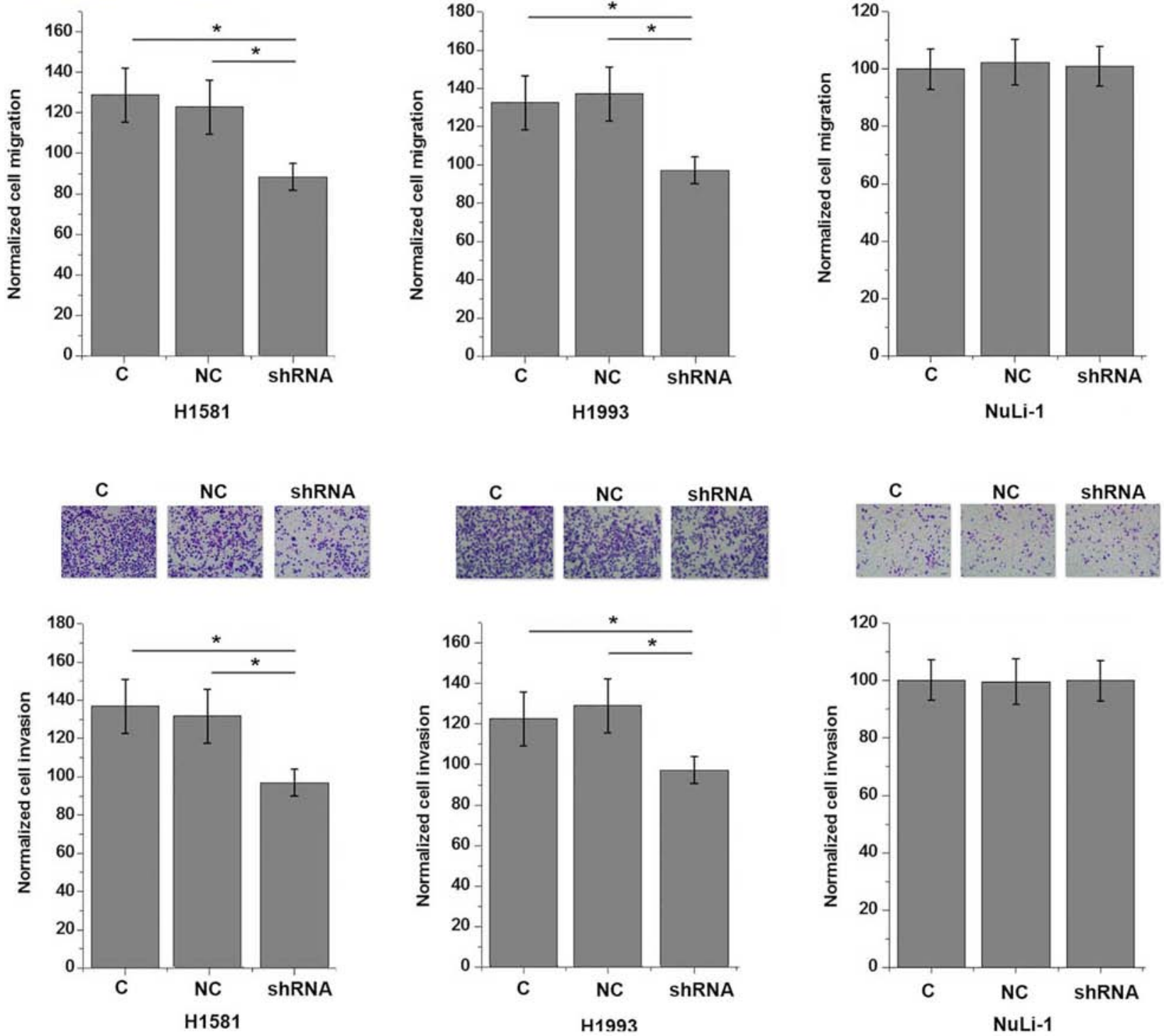

Figure 6. Effects of AWPPH shRNA-knockdown on cell migration and invasion. Effects of AWPPH shRNA knockdown treatment on (A) migration and (B) invasion of cells of normal human lung cell line NuLi-1 and human non-small cell lung cancer cell lines H1581 and H1993. Magnification X40. ${ }^{*}<<0.05 . \mathrm{C}$, control cells without any treatment; NC, cells transfected with scrambled shControl; sh, short hairpin.

lncRNAs have been suggested to participate in the whole procedure of the development of NSCLC (16-18) and therefore may not be able to predict a specific stage of NSCLC, such as tumor metastasis.

Functionality of lncRNA AWPPH has been characterized in hepatocellular carcinoma (11), bladder cancer (12) and triple negative breast cancer (13). AWPPH was upregulated in hepatocellular carcinoma and overexpression of AWPPH, not only promoted hepatocellular carcinoma cell proliferation and migration in vitro, but also accelerated tumor growth and metastasis in vivo (11). A previous study that focused on the involvement of AWPPH in bladder cancer also showed that this IncRNA may participate in both tumor growth and metastasis (12). However, this study observed no significant differences in AWPPH expression levels among patients with NSCLC with different tumor sizes, even after patients were grouped according to largest diameter. In contrast, all patients with different types of distant tumor metastasis showed upregulated expression of AWPPH compared with patients with non-tumor metastasis. The in vitro cell migration and invasion assay showed that AWPPH expression is an enhancer of NSCLC cell migration and invasion. These results suggest that, instead of participating in the entire process of NSCLC, AWPPH may only serve an important role in metastasis of NSCLC, or that metastasis of NSCLC induces the upregulation of AWPPH.

An increasing number of studies have confirmed that TGF- $\beta$ is the master regulator of epithelial mesenchymal transition (20), which is the key marker of the progression of metastasis in 
different malignancies (21). Consistently, this study also observed reduced migration and invasion abilities of NSCLC cells after TGF- $\beta$ inhibitor treatment. This study indicated that AWPPH is likely an upstream regulator of TGF- $\beta 1$, due to the fact that AWPPH overexpression upregulated TGF- $\beta 1$. The addition of exogenous TGF- $\beta 1$ however, did not affect AWPPH, and TGF- $\beta 1$ inhibition attenuated the effects of AWPPH overexpression on NSCLC cell behavior. However, these data only supported an AWPPH-TGF- $\beta 1$ sequential signaling transduction. Whether this regulation is direct or indirect remains unclear. AWPPH is host to MIR4435-2, while the function of MIR4435-2, to the best of our knowledge, has yet to be revealed. Further studies investigating whether MIR4435-2 may mediate the interaction between AWPPH and TGF- $\beta 1$ are to be performed.

In addition, this study indicated that AWPPH overexpression showed no significant effect on the biological behaviors of cells of normal human lung cell line NuLi-1. Therefore, AWPPH may serve as a safe target for the prevention and treatment of NSCLC.In conclusion, AWPPH is overexpressed in NSCLC. Overexpression of AWPPH promotes the migration and invasion of NSCLC cells possibly through the activation of TGF- $\beta$ signaling.

\section{Acknowledgements}

Not applicable.

\section{Funding}

No funding received.

\section{Availability of data and materials}

The datasets used and/or analyzed during the current study are available from the corresponding author on reasonable request.

\section{Authors' contributions}

YH and AL designed experiments. YH, HA and ZW performed experiments. YH and ZW analyzed data. AL drafted the manuscript. All authors have read and approved the manuscript.

\section{Ethics approval and consent to participate}

The Ethics Committee of Affiliated Xing Tai People Hospital of Hebei Medical University approved the present study and all participants provided written informed consent.

\section{Patient consent for publication}

Not applicable.

\section{Competing interests}

The authors declare that they have no competing interests.

\section{References}

1. Bray F, Ferlay J, Soerjomataram I, Siegel RL, Torre LA and Jemal A: Global cancer statistics 2018: GLOBOCAN estimates of incidence and mortality worldwide for 36 cancers in 185 countries. CA Cancer J Clin 68: 394-424, 2018.
2. Gridelli C, Rossi A, Carbone DP, Guarize J, Karachaliou N, Mok T, Petrella F, Spaggiari L and Rosell R: Non-small-cell lung cancer. Nat Rev Dis Primers 1: 15009, 2015.

3. Yeo CD and Joo H: P1.01-011 Roflumilast Attenuates Benzo(a) Pyrene-Induced Lung Cancer via Suppression of Airway Inflammation in Murine Model. J Thorac Oncol 12 (Suppl): S454, 2017.

4. Chen W, Zheng R, Baade PD, Zhang S, Zeng H, Bray F, Jemal A, Yu XQ and He J: Cancer statistics in China, 2015. CA Cancer J Clin 66: 115-132, 2016.

5. Temel JS, Greer JA, Muzikansky A, Gallagher ER, Admane S, Jackson VA, Dahlin CM, Blinderman CD, Jacobsen J, Pirl WF, et al: Early palliative care for patients with metastatic non-small-cell lung cancer. N Engl J Med 363: 733-742, 2010.

6. Cao M, Seike M, Soeno C, Mizutani H, Kitamura K, Minegishi Y, Noro R, Yoshimura A, Cai L and Gemma A: MiR-23a regulates TGF- $\beta$-induced epithelial-mesenchymal transition by targeting E-cadherin in lung cancer cells. Int J Oncol 41: 869-875, 2012.

7. Zhang J, Wang J, Zhao F, Liu Q, Jiang $\mathrm{K}$ and Yang GH: MicroRNA-21 (miR-21) represses tumor suppressor PTEN and promotes growth and invasion in non-small cell lung cancer (NSCLC). Clin Chim Acta 411: 846-852, 2010.

8. Xiao D and He J: Epithelial mesenchymal transition and lung cancer. J Thorac Dis 2: 154-159, 2010.

9. Gutschner T and Diederichs S: The hallmarks of cancer: A long non-coding RNA point of view. RNA Biol 9: 703-719, 2012.

10. Yuan JH, Yang F, Wang F, Ma JZ, Guo YJ, Tao QF, Liu F, Pan W, Wang TT, Zhou CC, et al: A long noncoding RNA activated by TGF- $\beta$ promotes the invasion-metastasis cascade in hepatocellular carcinoma. Cancer Cell 25: 666-681, 2014.

11. Zhao X, Liu Y and Yu S: Long noncoding RNA AWPPH promotes hepatocellular carcinoma progression through YBX1 and serves as a prognostic biomarker. Biochim Biophys Acta Mol Basis Dis 1863: 1805-1816, 2017.

12. Zhu F, Zhang X, Yu Q, Han G, Diao F, Wu C and Zhang Y: LncRNA AWPPH inhibits SMAD4 via EZH2 to regulate bladder cancer progression. J Cell Biochem 119: 4496-4505, 2018.

13. Wang K, Li X, Song C and Li M: LncRNA AWPPH promotes the growth of triple-negative breast cancer by up-regulating frizzled homolog 7 (FZD7). Biosci Rep 28: pii: BSR20181223, 2018.

14. Livak KJ and Schmittgen TD: Analysis of relative gene expression data using real-time quantitative PCR and the 2(-Delta Delta C(T)) method. Methods 25: 402-408, 2001.

15. Scheffler M, Bos M, Gardizi M, König K, Michels S, Fassunke J, Heydt C, Künstlinger H, Ihle M, Ueckeroth F, et al: PIK3CA mutations in non-small cell lung cancer (NSCLC): Genetic heterogeneity, prognostic impact and incidence of prior malignancies. Oncotarget 6: 1315-1326, 2015.

16. Pikor LA, Ramnarine VR, Lam S and Lam WL: Genetic alterations defining NSCLC subtypes and their therapeutic implications. Lung Cance 82: 179-189, 2013.

17. Cheng N, Li X, Zhao C, Ren S, Chen X, Cai W, Zhao M, Zhang Y, Li J, Wang $Q$ and Zhou C: Microarray expression profile of long non-coding RNAs in EGFR-TKIs resistance of human non-small cell lung cancer. Oncol Rep 33: 833-839, 2015.

18. Yang YR, Zang SZ, Zhong CL, Li YX, Zhao SS and Feng XJ: Increased expression of the lncRNA PVT1 promotes tumorigenesis in non-small cell lung cancer. Int J Clin Exp Pathol 7: 6929-6935, 2014

19. Lu KH, Li W, Liu XH, Sun M, Zhang ML, Wu WQ, Xie WP and Hou YY: Long non-coding RNA MEG3 inhibits NSCLC cells proliferation and induces apoptosis by affecting p53 expression. BMC Cancer 13: 461, 2013.

20. Katsuno Y, Lamouille S and Derynck R: TGF- $\beta$ signaling and epithelial-mesenchymal transition in cancer progression. Curr Opin Oncol 25: 76-84, 2013.

21. Micalizzi DS, Farabaugh SM and Ford HL: Epithelial-mesenchymal transition in cancer: Parallels between normal development and tumor progression. J Mammary Gland Biol Neoplasia 15: 117-134, 2010.

This work is licensed under a Creative Commons Attribution-NonCommercial-NoDerivatives 4.0 International (CC BY-NC-ND 4.0) License. 